\title{
Novel Technologies Can Provide Effective Dressing and Securement for Peripheral Arterial Catheters: A Pilot Randomised Controlled Trial in the Operating Theatre and the Intensive Care Unit
}

\begin{abstract}
Background: Peripheral arterial catheters are widely used in the care of intensive care patients for continuous blood pressure monitoring and blood sampling, yet failure - from dislodgement, accidental removal, and complications of phlebitis, pain, occlusion and infection - is common. Whilst appropriate methods of dressing and securement are required to reduce these complications that cause failure, few studies have been conducted in this area
\end{abstract}

Objectives: To determine initial effectiveness of one dressing and two securement methods versus usual care, in minimising failure in peripheral arterial catheters. Feasibility objectives were considered successful if 90/120 patients (75\%) received the study intervention and protocol correctly, and had ease and satisfaction scores for the study dressing and securement devices of $\geq 7$ on Numerical Rating Scale scores 1-10.

Methods: In this single-site, four-arm, parallel, pilot randomised controlled trial, patients with arterial catheters, inserted in the operating theatre and admitted to the intensive care unit postoperatively, were randomly assigned to either one of three treatment groups (bordered polyurethane dressing $(\mathrm{n}=30)$; a sutureless securement device $(\mathrm{n}=31)$; tissue adhesive $(\mathrm{n}=32)$ ), or a control group (usual practice polyurethane dressing (not bordered) $(\mathrm{n}=30)$ ).

Results: One hundred and twenty-three patients completed the trial. The primary outcome of catheter failure was 2/32 (6.3\%) for tissue adhesive, 4/30 (13.3\%) for bordered polyurethane, 5/31 (16.1\%) for the sutureless securement device, and 6/30 (20\%) for the control usual care polyurethane. Feasibility criteria were fulfilled. Cost analysis suggested that tissue adhesive was the most cost effective. 
Conclusions: The pilot trial showed that the novel technologies were at least as effective as the present method of a polyurethane dressing for dressing and securement of arterial catheters, and may be cost effective. The trial also provided evidence that a larger, multicentre trial would be feasible.

Key Words: peripheral arterial catheters; dressings; securement; nursing; anaesthesia; critical care; randomised controlled trial; evidence-based practice 


\section{Introduction}

Peripheral arterial catheters are widely used in the care of critically ill patients. They are a vital component of contemporary management of patients in the operating theatre (OT) and intensive care unit (ICU), and are usually inserted into a peripheral artery for continuous blood pressure monitoring and blood sampling for frequent blood gas analysis. Worldwide annual usage of arterial catheters is extensive, and is reported as up to eight million in the USA, and 2.5 million in Europe.(Gowardman, Lipman, \& Rickard, 2010; Scheer, Perel, \& Pfeiffer, 2002) Arterial catheters can fail before the completion of treatment due to complications of accidental removal, partial/complete dislodgement, occlusion, pain, phlebitis and infection, which may be either local or catheter-related. Catheter-related blood stream infections (CRBSI) incur hospital costs of \$US 1.2 million annually in the USA,(Centres for Disease Control (CDC), 2011) and increase patients’ length of hospital stay.(Dimick et al., 2001; Warren et al., 2006) The insertion site of an arterial catheter is usually dressed with a commercially produced transparent dressing, which assists in maintaining the catheter's position and plays a role in the prevention of microbial entry to the wound. Catheter failure incidence in peripheral arterial catheters is not often reported in the literature, but the few studies available suggest that up to 69\% (40/58) of arterial catheter insertion incidents are related to inadequate securement, and 24\% (60/249) of catheter use problems involved dislodgement or inadvertent removal.(Durie, Beckmann, \& Gillies, 2002) Further, high rates of accidental removal of arterial catheters have been described compared with accidental removal of central venous catheters in intensive care studies, with twice as many,(Lorente, Huidobro, Martin, Jimenez, \& Mora, 2004) and four times as many reported.(Carrión et al., 2000) Other literature acknowledges the importance of infection in peripheral arterial catheters, which also causes catheter failure. The incidence of arterial catheter-related infection in intensive care has been reported as 0.59 per 1,000 catheter days, with 0.34\% developing CRBSI,(Lorente, Santacreu, Martin, Jemenez, \& Mora, 2006) and point prevalence rates stating $0.8 \%$ and 1.7 per 1,000 catheter days.(Maki, Kluger, \& Crnich, 2006) Systematic review and meta-analysis have confirmed and consolidated impressions that arterial catheters may have a 
substantial burden of CRBSI, with pooled incidence of CRBSI in arterial catheters reporting a rate of 0.96 per 1,000 catheter days.(O'Horo, Maki, Krupp, \& Safdar, 2014)

Inadequate peripheral intravascular catheter securement remains a poorly researched area of patient care, and has been identified as a priority for improvement.(Schears, 2006) There is a paucity of quality studies reporting efficacy of dressing and securement methods for peripheral arterial catheters, with only one previous study (not randomised),(Stephenson, 2005) and a recent pilot randomised controlled study in cardiac surgical intensive care patients.(Edwards et al., 2014) Specialty anaesthetic and ICU nurses are largely responsible for post-insertion care of arterial catheters, in particular dressings and securement, and play a pivotal role in preventing the catheter-related complication of failure, including premature catheter removal.

\section{Dressing/securement methods}

The current Guidelines by the Centres for Disease Control (CDC) recommend covering the peripheral arterial catheter site with sterile gauze or a sterile, transparent, semipermeable dressing.(O'Grady et al., 2011) A sutureless securement device (SSD) is the specified recommended method for securement of the catheter instead of sutures, in order to reduce the risk of infection and needlestick injury.(O'Grady et al., 2011) Different dressings are available for use over the arterial catheter site. They are small and large transparent, semipermeable dressings, termed in this trial as usual care polyurethane, and include Tegaderm ${ }^{\mathrm{TM}}\left(3 \mathrm{M}^{\mathrm{TM}}\right)$ and Opsite ${ }^{\circledR}$.(Smith \& Nephew) A more recent version of transparent dressing involving novel technology to enhance adhesion and including a reinforced opaque adhesive border is Tegaderm ${ }^{\mathrm{TM}}$ I.V. Advanced,(3M $\left.{ }^{\mathrm{TM}}\right)$ referred to in this study as Bordered Polyurethane (BPU). Traditionally, these dressings have been used in conjunction with adhesive tape to secure the arterial catheter tubing. An alternative to tape is a precision made SSD, specifically used with arterial catheters, such as the novel approaches of the StatLock ${ }^{\circledR}$ arterial stabilisation device $\left(\operatorname{Bard}{ }^{\circledR}, 2013\right)$ or the Grip-Lok ${ }^{\circledR}$ device.(Zefon) Transparent dressings, tapes, and SSDs, which are used for arterial catheters, 
are also used for IV catheters. Skin glue, also termed tissue adhesive (TA), has had novel use in a limited capacity for the securement of intravascular catheters, providing another alternative to sutures or a SSD. There have been a few small reports of the use of Histoacryl ${ }^{\circledR}$ TA to secure central venous catheters and epidural catheters in the UK.(Wilkinson \& Chikhani, 2007; Wilkinson \& Fitz-Henry; Wilkinson, Sheikh, \& Jayamaha) The effectiveness of the use of an SSD in arterial catheters has been reported,(Stephenson, 2005) and recent pilot work on the novel dressing and securement technologies of BPU, an SSD, and TA for arterial catheters, has been performed to inform of effectiveness and the feasibility of further study.(Edwards et al., 2014)

\section{Study aims}

We aimed to determine and compare initial effectiveness of a BPU, SSD and TA versus usual care, in preventing failure in peripheral arterial catheters inserted in the operating theatre and cared for in the intensive care unit, as well as their suitability for study in a large multi-centre randomised trial. We modelled our approach on a previous pilot study, which set feasibility criteria to determine success.(Cook et al., 2005)

\section{Methods}

Study design, setting and participants

This pilot, single-site, four-arm, parallel, randomised controlled trial was conducted within the OT complex and the Department of Intensive Care Medicine at Royal Brisbane and Women’s Hospital in Queensland, Australia. The Principal Investigator screened all surgical patients booked for post-operative ICU admission at the anaesthetic Pre-Admissions Clinic and inpatients, Monday to Friday, from September 1, 2012 to March 28, 2013. Patient inclusion criteria were: aged at least 18 years; scheduled elective major surgery requiring an arterial catheter; and booked ICU post-operative care. Patients were excluded if: they had known allergies to the study products; were non-English speaking and an interpreter 
was not available; the arterial catheter was to be inserted through burned, diseased, or damaged skin; and they were diaphoretic.

Since the primary aim of this pilot trial encompassed feasibility rather than hypothesis testing, formal power calculations were not appropriate. Thus, a sample size of $n=120$, with three intervention groups and one control group, each $n=30$, was selected. Such group sizes were used to achieve at least a $68 \%$ confidence of an accurate estimate of arterial catheter failure within a $7 \%$ margin of risk for inaccuracy.(Hertzog, 2008) They were also realistic regarding time and cost, and would yield confidence intervals with lower limits to assist in the definition of a range of values for later power analysis.(Hertzog, 2008)

The university and hospital human research and ethics committees approved the protocol, and written, informed consent from all participants was obtained prior to enrolment.

\section{Randomisation and concealment}

Random allocation of patients was undertaken by the Principal Investigator via a computergenerated system of centralised web-based randomisation. Patients were randomly assigned on the day of surgery to one of the three treatment groups or the control group. Block randomisation and stratified blocks were used, with randomly varied block sizes. Allocation concealment was achieved until the point of study entry, after which, due to the nature of the intervention, it was not possible to blind patients or clinical staff. Laboratory staff were blinded for assessment of microbiological endpoints.

\section{Study interventions}

Group One was the BPU (not sutured) (Tegaderm ${ }^{\mathrm{TM}}$ I.V. Advanced Securement Dressing 1683, 3M $\mathrm{M}^{\mathrm{TM}}$, St Paul); Group Two was the SSD and a small polyurethane dressing over the catheter insertion 
site (StatLock ${ }^{\circledR}$ Arterial Select Stabilisation Device, Bard ${ }^{\circledR}$, Salt Lake City); and Group Three was TA (Histoacryl ${ }^{\circledR}$, B Braun, Bella Vista) and a small polyurethane dressing over the catheter insertion site. The control group had arterial catheters that were not sutured, and were secured with usual care polyurethane (Tegaderm ${ }^{\mathrm{TM}} 1624 \mathrm{~W}, 3 \mathrm{M}^{\mathrm{TM}}$, St Paul).

\section{Outcomes measures}

The primary outcome was peripheral arterial catheter failure, which was a composite of one or more of complete arterial catheter dislodgement, occlusion, phlebitis, and infection, either local or CRBSI. Each of these failure types was considered on an individual basis for a secondary outcome. In addition to peripheral arterial catheter dwell time, the costs, workload, ease of dressing/device application, and patient/staff satisfaction were included as secondary outcomes.

The Principal Investigator collected data using a standardised data collection tool developed for use on a personal laptop computer. A paper data collection form mirrored some of the computer-based data points and was kept at the bedside for convenient documentation by ICU nurses. Data were collected on insertion of the arterial catheter in the OT, at each ICU patient's bedside daily, and on ICU discharge. Other points of data collection were at the time of dressing adjustment or changes, at catheter removal, and final assessment at 48 hours post catheter removal. The reasons for catheter removal were documented as: completed therapy (not failed); blocked; could not aspirate; monitor failure; accidental dislodgement; and/or signs of infection.

\section{Statistical analyses}

Data were exported from the Microsoft Access 2010 database into Stata 12.1(StataCorp LP) for cleaning and analysis. The statistical analyses of the pilot work were mainly descriptive, with an Intention 
to Treat analysis framework assumed, and patient flow following the Consolidated Standards of Reporting Trials (CONSORT) Guidelines.(Altman et al., 2001)

Mean values and standard deviations were reported for normally distributed data, while median values and the $25 \%$ and $75 \%$ percentiles were reported otherwise. The Kaplan-Meier curve was used to show the cumulative survival probability of arterial catheters over time. Arterial catheter failure over time was tested with the log-rank test for equality of survivor functions, comparing the three alternative interventions with the control group. Univariable and multivariable Cox proportional hazards regression models were used to assess for independent relationships between the potential explanatory variables for catheter failure - group, age, gender, skin type, comorbidities, smoker, dominant side, health care professional who inserted the catheter, number of insertion attempts, sterile gloves worn, number of staff and which staff members applied the dressing/securement device, ease of application, time to apply, or number of securing devices - and the dichotomous outcome of failure. The incident rate of catheter failure was expressed as the number of catheter failures per 1,000 catheter hours. For the purposes of this study, feasibility of a larger trial was indicated if a Numerical Rating Score (NRS) score of $\geq 7$ was achieved for more than 90/120 (75\%) of patients (90\% CI) in all of the categories of ease of application, satisfaction on removal for staff, and satisfaction on removal and overall satisfaction for patients.

Research nurse workload was assessed by recording the total time taken to carry out all research tasks. The rate of recruitment, number of eligible patients who agreed to participate, number of patients who received the correct protocol, and completed the trial, were also recorded.

Cost effectiveness was determined by setting a threshold amount that society would be willing to pay for an incremental health gain: set at \$AUD 100, which was the cost to replace an arterial catheter. The effect outcome was the probability of catheter success. The economic concept of the net monetary benefit approach was used to perform the analysis. The net benefit, which was calculated in terms of money with a ceiling ratio that was considered acceptable to pay, was estimated for the four catheter dressings/securements. The preferred option was the one with the maximum average net benefit.(Tuffaha, 
Gordon, \& Scuffham, 2014) Further, value of information analysis was used to estimate the value of additional research and to inform optimal trial design.(Claxton \& Posnett, 1996; Eckermann, Karnon, \& Willan, 2010; 2005)

\section{Results}

From a total of $n=150$ patients screened for eligibility, $n=132$ were randomised, and $n=123$

completed the trial (see Figure 1). The demographic and clinical profiles of patients in all groups were similar at baseline for most variables (see Table 1). Small imbalances were not considered to be clinically important and were most likely due to chance in accordance with a rigorous randomisation procedure. Thus, statistical tests to compare the balance of baseline differences were not performed in compliance with recommendations in the CONSORT statement (2010) and other literature (Altman, 1985; Altman \& Dore, 1990; Burgess, Gebski, \& Keech, 2003; Moher et al., 2010; Senn, 1995). 
Intervention effect on primary and secondary outcomes

The primary outcome of arterial catheter failure prior to completion of therapy was 6/30 (20\%) for control polyurethane, compared with 4/30 (13.3\%) for BPU, 5/31 (16.1\%) for SSD, and 2/32 (6.3\%) for TA. The effect size of TA was an absolute reduction of $14 \%$ compared to controls, with absolute reductions of $6.7 \%$ and $3.9 \%$ for BPU and SSD respectively. Table 2 provides details on the mechanism of failure by group.

The control catheter failure rate was 7.94 per 1,000 arterial catheter hours, (95\% CI, 3.57- 17.68). The catheter failure rates for the interventions were: BPU group, 5.09 per 1,000 catheter hours (95\% CI, 1.91 - 13.56, $p=0.51$ ); SSD group, 6.03 per 1,000 catheter hours (95\% CI, $2.51-14.48, p=0.66)$; and TA group, 2.33 per 1,000 catheter hours (95\% CI, 0.58 - 9.31, $p=0.13$ ) (see Figure 2). There were no statistically significant differences in outcomes of the study groups compared to controls. The secondary outcomes of individual types of failure and catheter dwell times were not significantly different in the treatment or control groups (see Table 2). No infection outcomes were observed in the study.

Compared with the control group, the hazard ratio of catheter failure was 0.32 (95\% CI, 0.06 1.66) in the TA group, 0.64 (95\% CI, 0.17 - 2.44) in the BPU group, and 0.78 (95\% CI, $0.22-2.70$ ) in the SSD group. None were statistically significant. Significant predictors of catheter failure in the multivariate model were current smoker and female $(p<0.05)$ (see Table 3).

Kaplan-Meier survival curves for the three treatment groups and the control were plotted to display failure of arterial catheters over time (see supplementary material). There was little difference between curves up until the average dwell time at the 24-hour mark, after which the TA group had the highest proportion in use of the three interventions (non-significant $p=0.562$ ). There were seven arterial catheters in place at 48 hours. 
Feasibility

The percentage of eligible people who agreed to participate was $100 \%$, and the average recruitment was 21 patients per month. Of the 146 patients recruited, 132 (90.4\%) were randomised, and timely performance of the protocol occurred for 123 (93.2\%) (see Figure 1). Satisfaction and ease of application NRS scores were skewed, so they were transformed into dichotomous data for comparison of proportions in each group rating $\geq 7$ for each item. TA and SSD had statistically worse scores for staff ease of application compared to controls, $25(78.1 \%)$ and $22(71.0 \%)$ respectively, (both $p<0.05)$. However, TA still met the feasibility cut-off for easy application of $75 \%$, with $78 \%$ of ratings being $\geq 7$. For the other ratings for staff ease of removal, patient ease of removal, and overall satisfaction, there were no significant differences between groups and controls (all $p>0.05$ ). All groups met the feasibility criteria for patient removal ratings and overall patient satisfaction. The percentages of patient satisfaction with removal ratings $\geq 7$ by group were $100 \%$ for BPU, SPU, and TA, and $93 \%$ for SPU control. The percentages of patient overall satisfaction ratings of $\geq 7$ by groups were: $86.7 \%$ for BPU; $96.8 \%$ for SSD; 96.9\% for TA; and $96.4 \%$ for SPU control. Other feasible NRS scores of $\geq 75 \%$ were staff ease of application for BPU at $93.3 \%$ and control SPU at 100\%, as well as staff ease of removal for BPU at 86.7\%, and TA at $84.4 \%$.

\section{Workload of research activities}

The total time taken to carry out pre-protocol implementation research activities, (before randomisation of the first patient) was 151 hours. The Principal Investigator's daily research tasks were timed and collated for 12 consecutive days (Monday to Friday), with the mean total time per day for research tasks being $5.2(1.67)$ hours. 


\section{Cost-effectiveness}

With the willingness to pay threshold set at \$AUD 100 to gain the desirable outcome of catheter success, the average net benefit was highest for tissue adhesive (\$AUD 14.10), with a probability of 35\%. A four-arm trial design was found to provide the highest net benefit, and was thus the most economical design for a large trial.

\section{Discussion}

The results from this pilot trial showed that the new dressing and securement technologies were clinically effective in preventing arterial catheter failure when compared with usual care. Further, it was shown that it would be feasible to undertake further study of the dressing and securement of peripheral arterial catheters with the interventions BPU, SSD and TA. It is encouraging for future studies that all three tested technologies had lower absolute incidence of arterial catheter failure than the control catheters, thus suggesting that all three interventions may reduce arterial catheter failure. However, regardless of group, the overall study failure rate was 17/123 (13.8\%) for this vital and ubiquitous medical device; a high rate, especially given that devices were only required for a short (approximately 24 hour) period. The study of arterial catheter failure rates does not command discussion in the current literature and, thus, this study is extremely important.

BPU dressings have not been well researched, with no previous randomised controlled trials to investigate the manufacturer's indications that BPU dressings minimise the risks of dislodgement and increase securement. This pilot study showed an accidental removal incidence of 2/30 (6.7\%), which seemed to indicate a lack of suitability compared with the other interventions, which had no accidental removals. BPU dressings may have been more satisfying (presumably easier) to remove for nurses, than the controls $(86.7 \%$ vs $65.5 \%$ scored $\geq 7$ out of $10, p=0.072)$. These results are difficult to interpret, and 
may be coincidental findings, since the reinforced picture-frame design of the BPU would appear to require more work for nurses to remove.

Several studies in IV catheters have shown superior performance of the StatLock® SSD over usual care polyurethane dressings or tape.(Frey \& Schears, 2006; Royer, 2003; Schears; 2006) There has only been one large prior study of StatLock ${ }^{\circledR}$ in arterial catheters.(Stephenson, 2005) In our study, the SSD catheter failure incidence was 5/31 (16.1\%), which was not significantly different to incidence in the control group. In Stephenson's study of 995 patients the SSD failure incidence was 12.8\%.(Stephenson, 2005) This is not largely dissimilar to the observed SSD failure of $16.1 \%$ in the current study, thus supporting the validity of the findings. Stephenson(Stephenson, 2005) reported a lower incidence of failure in the SSD group than in their control group (25\%) as statistically significant, while our study failed to find the same difference. However, we had the advantage of a robust randomised design, adding to the quality of the body of knowledge on the SSD approach.

There were only 22 (71\%) of SSD patients whose NRS scores were $\geq 7$ for ease of application, compared with $100 \%$ in the control group, and this difference was statistically significant at $p=0.002$. This score was lower than our feasibility criteria of $75 \%$ of scores being $\geq 7$. Thus, staff clearly found application less easy. This SSD required a multistep procedure to apply, with the first step to prepare the skin, prior to applying the adhesive pads, and then the arterial catheter and tubing to be inserted into the SSD itself. This likely contributed to a longer application time compared with the other interventions, and led to lower satisfaction ratings for application. In contrast, rating for satisfaction with removal for patients and staff, and for overall satisfaction, were similar for patients in the SSD and control groups, and these results were feasible. Further education and experience with the SSD may increase the ease of application ratings.

The use of skin glue (TA) was a novel inclusion in this trial in response to concerns in the literature regarding lack of effective securement of arterial catheters,(Carrión et al., 2000; Durie et al., 2002) and the potential risk of infection which may be underestimated.(Koh, Gowardman, Rickard, 
Robertson, \& Brown, 2008; Lucet et al., 2010; Traore, Liotier, \& Souweine, 2005; Wittekamp et al., 2013) The glue was associated with the lowest number of arterial catheter failures as the primary outcome, at 2/32 (6.3\%), however, this was not statistically different, $p=0.14$, in comparison with controls. There were two patients with TA who remained in ICU for four days, and their arterial catheters were secured successfully. If effectiveness could be confirmed in a larger trial, this would be of great benefit. The economic evaluation also showed that the new method of TA was most cost effective of the three interventions in comparison with control polyurethane, indicating that it was the preferred intervention, and demonstrating its potential further.

In terms of planning future research, on the basis of this pilot study, comparing the observed proportions with 90\% power and $p=0.05$ (two-sided),(University of British Colombia, 2014) a future trial would need group sizes of 983 patients for SSDs (16\% failure), 589 for BPU (13\% failure) and 61 patients TA patients (14\% failure). As regards to feasibility indicators, all patients (100\%) who were assessed as eligible gave written informed consent to participate in the trial, suggesting the process was effective. Mean daily work time for the research nurse for a 12-day period was 5.2 (1.6) hours. Therefore, the estimated daily research nurse requirements for the larger trial were approximately 5.5 to 8 hours per day, Monday to Friday, with the recommended work hours between 06:00 and 16:30. A senior research nurse and up to eight research nurses would be required full-time for eight months.

\section{Limitations}

This pilot trial, due to the absence of a known effect size, was not sufficiently powered to detect statistical significances. It was also limited by it being a single site trial involving only short-term arterial catheters. 


\section{Conclusion}

This pilot research makes an original contribution to knowledge by providing initial information on the effectiveness of the tested novel technologies to secure arterial catheters, together with a thorough investigation of pilot methodology to verify the feasibility of future extensive use of these products in randomised testing. The trial has contributed to optimising care of patients with peripheral arterial catheters, which has previously attracted little research attention.

Word count: 3496/3500

\section{Acknowledgements}

The authors express their appreciation and sincerely thank the trial participants and the anaesthetic and intensive care staff who made the trial possible.

\section{Conflict of Interests}

There are no conflicts of interests.

\section{Funding}

This research received no specific grant from any funding agency in the public, commercial or not-for-profit sectors. Funding was provided for products by the Alliance for Vascular Access Teaching and Research Group (AVATAR) at Griffith University. 


\section{References}

3M ${ }^{\mathrm{TM}}$, St Paul. (2013a). Tegaderm ${ }^{\mathrm{TM}}$ I.V. Advanced securement dressings: Designed for comfort and securement. Retrieved October 10, 2013, from http://multimedia.3m.com/mws/mediawebserver?mwsId=66666UgxGCuNyXTtn8T6NxfaEVtQE cuZgVs6EVs6E666666--\&fn=70-2010-8039-0.pdf

3M ${ }^{\mathrm{TM}}$, St Paul. (2013b). Tegaderm ${ }^{\mathrm{TM}}$ transparent film dressings product profile. Retrieved December 10, 2013, from http://multimedia.3m.com/mws/mediawebserver?mwsId=66666UgxGCuNyXTtmxTc58TXEVtQ EcuZgVs6EVs6E666666--\&fn=70-2008-8759-7\%20Tegaderm\%20Transpar

Altman, D. G. (1985). Comparability of randomised groups. Journal of the Royal Statistical Society. Series D (The Statistician), 34(1), 125-136.

Altman, D. G., \& Dore, C. J. (1990). Randomisation and baseline characteristics in clinical trials. The Lancet, 335, 149-153.

Altman, D. G., Schultz, K. F., Moher, D., Egger, M., Davidoff, F., Elbourne, D., . . Lang, T. (2001). The revised CONSORT statement for reporting randomized trials: Explanation and elaboration. Annals of Internal Medicine, 134(8), 663-694.

Bard ${ }^{\circledR}$, Salt Lake City. (2013). Bard® Access Systems stabilization devices: StatLock ${ }^{\circledR}$ Arterial Select Stabilisation Device. Retrieved October 10, 2013, from http://www.bardaccess.com/statlockarterial-select.php

Burgess, D. C., Gebski, V. J., \& Keech, A. C. (2003). Baseline data in clinical trials. Medical Journal of Australia, 179, 105-107.

Carrión, M. I., Ayuso, D., Marcos, M., Robles, M. P., De la Cal, M., \& Alía, I. (2000). Accidental removal of endotracheal and nasogastric tubes and intravascular catheters. Critical Care Medicine, 28(1), 63-66. 
Centres for Disease Control (CDC). (2011). National Health and Safety Network (NHSN). Retrieved May 14, 2012, from http://www.cdc.gov/nhsn/

Claxton, K., \& Posnett, J. (1996). An economic approach to clinical trial design and research prioritysetting. Health Economics, 5(6), 513-524.

Cook, D. J., Rocker, G., Meade, M., Guyatt, G., Geerts, W., Anderson, D., . . Crowther, M. (2005). Prophylaxis of thromboembolism in critical care (PROTECT) trial: A pilot study. Journal of Critical Care, 20(4), 364-372. doi: 10.1016/j.jcrc.2005.09.010

Dimick, J. B., Pelz, R. K., Consunji, R., Swoboda, S. M., Hendrix, C. W., \& Lipsett, P. A. (2001). Increased resource use associated with catheter-related blood stream infection in the surgical intensive care unit. Archives of Surgery, 136(229-234).

Durie, M., Beckmann, U., \& Gillies, D. M. (2002). Incidents relating to arterial cannulation as identified in 7525 reports submitted to the Australian Incident Monitoring Study (AIMS-ICU). Anaesthesia and Intensive Care, 30, 60-65.

Eckermann, S., Karnon, J., \& Willan, A. R. (2010). The value of value of information best informing research design and prioritization using current methods. Pharmacoeconomics, 28(9), 699-709.

Edwards, M., Rickard, C. M., Rapchuk, I., Corley, A., Marsh, N., Spooner, A. J., . . Fraser, J. F. (2014). A pilot trial of bordered polyurethane dressings, tissue adhesive and sutureless devices compared with standard polyurethane dressings for securing short-term arterial catheters. Critical Care and Resuscitation, 16(3), 175-183.

Frey, A. M., \& Schears, G. J. (2006). Why are we stuck on tape and suture? Journal of Infusion Nursing, 29(1), 34-38.

Gowardman, J. R., Lipman, J., \& Rickard, C. M. (2010). Assessment of peripheral arterial catheters as a source of sepsis in the critically ill: A narrative review. Journal of Hospital Infection, 75(1), 1218. 
Hertzog, M. A. (2008). Considerations in determining sample size for pilot studies. Research in Nursing \& Health, 31(2), 180-191. doi: 10.1002/nur.20247

Koh, D. B., Gowardman, J. R., Rickard, C. M., Robertson, I. K., \& Brown, A. (2008). Prospective study of peripheral arterial catheter infection and comparison with concurrently sited central venous catheters. Critical Care Medicine, 36(2), 397-402.

Lorente, L., Huidobro, M., Martin, M., Jimenez, A., \& Mora, M. (2004). Accidental catheter removal in critically ill patients: A prospective and observational study. Critical Care, 8(4), R229-R233.

Lorente, L., Santacreu, R., Martin, M., Jemenez, A., \& Mora, M. (2006). Arterial catheter-related infection of 2,949 catheters. Critical Care, 10(3), R.83. doi: 10.1186/cc4930

Lucet, J. C., Bouadma, L., Zahar, J. R., Schwebel, C., Geffroy, A., Pease, S., . . Timsit, J.-F. (2010). Infectious risk associated with arterial catheters compared with central venous catheters. Critical Care Medicine, 38(4), 1030-1035.

Maki, D. G., Kluger, D. M., \& Crnich, C. J. (2006). The risk of bloodstream infection in adults with different intravascular devices: A systematic review of 200 published prospective studies. Mayo Clinic Proceedings, 81(9), 1159-1171. doi: 10.4065/81.9.1159

Moher, D., Hopewell, S., Schulz, K. F., Montori, V. M., Gøtzsche, P. C., Devereaux, P. J., .. Altman, D. G. (2010). CONSORT 2010 Explanation and Elaboration: Updated guidelines for reporting parallel group randomised trials. [Journal Article].

O'Grady, N. P., Alexander, M., Burns, L. A., Dellinger, P., Garland, J., Heard, S. O., . . Saint, S. (2011). CDC Guidelines for the Prevention of Intravascular Catheter-Related Infections, 2011. Retrieved February 20, 2012, from http://www.cdc.gov/hicpac/BSI/BSI-guidelines-2011.html

O'Horo, J. C., Maki, D. G., Krupp, A. E., \& Safdar, N. (2014). Arterial catheters as a source of bloodstream infection: A systematic review and meta-analysis. Critical Care Medicine. doi: 10.1097/CCM.0000000000000166 
Royer, T. (2003). Improving short peripheral IV outcomes: A clinical trial of two securement methods. The Journal of the Association of Vascular Access, 8(4), 45-49.

Schears, G. J. (2006). Summary of product trials for 10,164 patients: Comparing an intravenous stabilizing device to tape. Journal of Infusion Nursing, 29(4), 225-231.

Scheer, B., Perel, A., \& Pfeiffer, U. (2002). Clinical review: Complication and risk factors of peripheral arterial catheters used for haemodynamic monitoring in anaesthesia and intensive care medicine. Critical Care, 6(3), 199-204.

Senn, S. (1995). Base logic: Tests of baseline balance in randomised clinical trials. Clinical Research and Regulatory Affairs, 12, 171-182.

Smith \& Nephew, North Ryde (2013). OPSITE® transparent waterproof film. Retrieved November 23, 2013, from http://www.smith-nephew.com/professional/products/all-products/opsite/

Smith, B. (2006). Peripheral intravenous catheter dwell times: A comparison of 3 securement methods for implementation of a 96-hour scheduled change protocol. Journal of Infusion Nursing, 29(1), 1417.

StataCorp LP, College Station, Texas, USA. (2014). Stata: Data analysis and statistical software. Retrieved February 25, 2014, from http://www.stata.com

Stephenson, C. (2005). The advantages of a precision-engineered securement device for fixation of arterial pressure-monitoring catheters: Clinical investigations. The Journal of the Association for Vascular Access, 10(3), 130-132.

Traore, O., Liotier, J., \& Souweine, B. (2005). Prospective study of arterial and central venous catheter colonization and of arterial and central venous catheter related bacteremia in intensive care units. Critical Care Medicine, 33(6), 1276-1280.

Tuffaha, H. W., Gordon, L. G., \& Scuffham, P. A. (2014). Value of information analysis in healthcare: a review of principles and applications. Journal of Medical Economics, 1-7. doi:10.3111/13696998.2014.907170 
University of British Colombia, Canada. (2014). Statistics: Power/Sample size calculator. Retrieved May 2, 2014, from http://www.stat.ubc.ca/ rollin/stats/ssize/n2.html

Warren, D. K., Quadir, W. W., Hollenbeak, C. S., Elward, A. M., Cox, M. J., \& Fraser, V. J. (2006). Attributable cost of catheter-associated infections among intensive care patients in a non-teaching hospital. Critical Care Medicine, 34(2084-2089).

Wilkinson, J. N., \& Chikhani, M. (2007). The use of Histoacryl ${ }^{\circledR}$ skin adhesive to secure thoracic epidural catheters: A volunteer study. Regional Anesthesia and Pain Medicine, 32(5 Supplement 1-5), 148.

Wilkinson, J. N., \& Fitz-Henry, J. (2008). Securing epidural catheters with Histoacryl ${ }^{\circledR}$ glue. Anaesthesia, 63(3), 324-324. doi: 10.1111/j.1365-2044.2008.05468.x

Wilkinson, J. N., Sheikh, N., \& Jayamaha, J. (2007). Tissue adhesive as an alternative to sutures for securing central venous catheters. Anaesthesia, 62(9), 969-970. doi: 10.1111/j.13652044.2007.05240.x

Willan, A. R., \& Pinto, E. M. (2005). The value of information and optimal clinical trial design. Statistics in Medicine, 24(12), 1791-1806.

Wittekamp, B., Chalabi, M., Van Mook, W., Winkens, B., Verbon, A., \& Bergans, D. (2013). Catheterrelated bloodstream infections: A prospective observational study of central venous and arterial catheters. Scandinavian Journal of Infectious Diseases, July 5th, 1-8. doi: doi:10.3109/00365548.2013.804632

Zefon, Ocala (2013). Grip-Lok ${ }^{\circledR} 4200$ ZAB: Arterial catheter securement. Retrieved November 13, 2013, from http://m.zefon.com/medical/pc/Securement-Products-c10.htm 